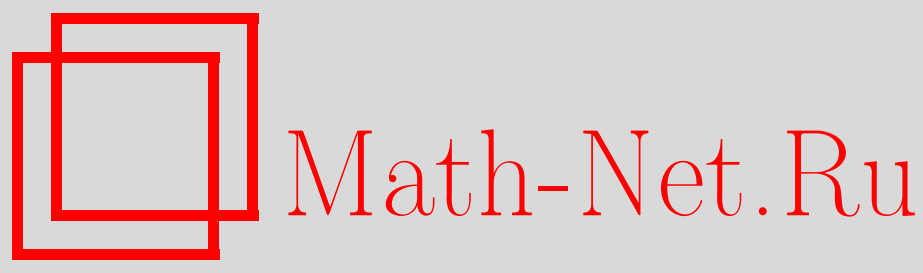

Х. Панахи, Л. Джахангири, Решения Родригеса уравнения Дирака для форминвариантных потенциалов: подход на основе мастер-функции, ТМФ, 2010, том 164, номер 2, 299-308

DOI: https://doi.org/10.4213/tmf6540

Использование Общероссийского математического портала Math-Net.Ru подразумевает, что вы прочитали и согласны с пользовательским соглашением http://www . mathnet.ru/rus/agreement

Параметры загрузки:

IP : 54.198 .187 .58

26 апреля 2023 г., 14:12:09

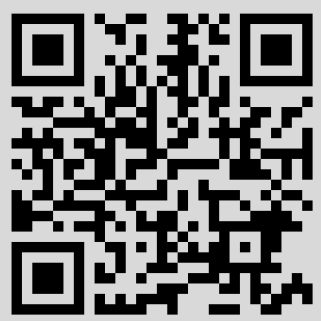




\title{
РЕШЕНИЯ РОДРИГЕСА УРАВНЕНИЯ ДИРАКА ДЛЯ ФОРМИНВАРИАНТНЫХ ПОТЕНЦИАЛОВ: ПОДХОД НА ОСНОВЕ МАСТЕР-ФУНКЦИИ
}

\begin{abstract}
Показано, что дифференциальное уравнение типа уравнения Шредингера для верхних спинорных компонент заряженного спинора в сферически-симметричном электромагнитном потенциале, выведенное из уравнения Дирака, можно преобразовать к уравнению Шредингера с некоторым форминвариантным потенциалом. Для электростатических потенциалов и релятивистских энергий различного вида путем ввода новых функций и замены переменных показано, что это уравнение преобразуется к дифференциальным уравнениям математической физики. Эти уравнения решаются с использованием мастер-функции, а спинорные волновые функции записываются в терминах многочленов Родригеса, ассоциированных с данными дифференциальными уравнениями.
\end{abstract}

Ключевые слова: уравнение Дирака, многочлен Родригеса, форминвариантный потенциал, подход на основе мастер-функции.

\section{1. ВВЕДЕНИЕ}

В последнее время идеи суперсимметрии и форминвариантности [1], [2] привлекли внимание исследователей и использовались в качестве алгебраических методов решения большого числа задач с нерелятивистскими форминвариантными потенциалами [3]. Решения уравнения Дирака с такими физическими потенциалами, как кулоновский потенциал [4], потенциал Морзе [5], потенциал гармонического осциллятора [6] и т.д., также рассматривались как релятивистские обобщения этих потенциалов. В работе [7] уравнение Дирака было решено для заряженного спинора в электромагнитном поле для сферически-симметричных потенциалов Дирака-Розена-Морзе, Дирака-Экарта, Дирака-Скарфа и Дирака-Пошля-Теллера. В этой работе путем решения дифференциального уравнения второго порядка типа уравнения Шредингера были найдены релятивистский спектр связанных состояний и спинорные волновые функции для верхней спинорной компоненты, а для нижней компоненты волновые функции получались путем действия линейного оператора

${ }^{*}$ Department of Physics, Guilan University, Rasht, Iran.

E-mail: t-panahi@guilan.ac.ir, laleh.jahangiry@yahoo.com 
первого порядка на верхнюю компоненту. С другой стороны, в работе [8] было показано, что стандартные дифференциальные уравнения второго порядка математической физики и связанные с ними дифференциальные уравнения обладают свойствами суперсимметрии и форминвариантной симметрии. В этой работе было также показано, что путем различного выбора многочлена не выше второй степени, называемого мастер-функцией, оператор дифференциального уравнения можно представить в виде произведения повышающих и понижающих операторов. Все известные одномерные форминвариантные потенциалы можно получить с использованием мастер-функции [9].

В настоящей работе подход на основе мастер-функции применяется для решения уравнения Дирака с некоторыми одномерными потенциалами. Предпринята попытка исследовать их форминвариантные симметрии и получить спинорные волновые функции в терминах многочленов Родригеса.

Работа построена следующим образом: в разделе 2 на основе результатов работы [7] путем применения унитарного преобразования уравнения Дирака со сферически-симметричным потенциалом строится дифференциальное уравнение второго порядка типа уравнения Шредингера для верхних спинорных компонент волновой функции. Кроме того, приводятся некоторые факты теории суперсимметрии и форминвариантных потенциалов в нерелятивистской квантовой механике. В разделе 3 показано, что полученное в разделе 2 дифференциальное уравнение второго порядка обладает форминвариантной симметрией в рамках формализма мастер-функции, а затем этот подход применяется для решения уравнения Дирака и определения электростатического потенциала и релятивистской энергии в терминах мастер-функции и весовой функции. В разделе 3 приводится пример, иллюстрирующий работу нашего метода, а также таблица решений уравнения Дирака для других потенциалов. Наконец, раздел 4 содержит краткое заключение.

\section{2. УРАВНЕНИЕ ДИРАКА СО СФЕРИЧЕСКИ-СИММЕТРИЧНЫМИ И ФОРМИНВАРИАНТНЫМИ ПОТЕНЦИАЛАМИ}

Будем следовать процедуре решения уравнения Дирака с форминвариантными потенциалами и сначала получим дифференциальное уравнение второго порядка типа уравнения Шредингера для верхних спинорных компонент из работы [7]. Положим $m=e=\hbar=1$ и $c=\alpha^{-1}$, где $\alpha$ - постоянная тонкой структуры. Тогда гамильтониан дираковской частицы в четырехкомпонентном электромагнитном потенциале $\left(A_{0}, \vec{A}\right)$ можно записать как

$$
H=\left(\begin{array}{cc}
1+\alpha A_{0} & -i \alpha \vec{\sigma} \cdot \vec{\nabla}+\alpha \vec{\sigma} \cdot \vec{A} \\
-i \alpha \vec{\sigma} \cdot \vec{\nabla}+\alpha \vec{\sigma} \cdot \vec{A} & -1+\alpha A_{0}
\end{array}\right),
$$

где $\vec{\sigma}-(2 \times 2)$-матрицы Паули. С учетом калибровочной инвариантности электромагнитный потенциал для статического сферически-симметричного распределения заряда имеет вид

$$
\left(A_{0}, \vec{A}\right)=(\alpha V(r), \hat{r} w(r)),
$$


где $\hat{r}$ - единичный вектор в радиальном направлении, $V(r)$ - электростатический потенциал, а $w(r)$ - калибровочное поле. Заменяя в двух недиагональных членах в уравнении (1) $\alpha \vec{\sigma} \cdot \vec{A}$ на $\pm i \alpha \vec{\sigma} \cdot \vec{A}$, из данного гамильтониана мы получаем двухкомпонентное радиальное уравнение Дирака [4]

$$
\left(\begin{array}{cc}
1+\alpha^{2} V(r) & \alpha\left[\frac{k}{r}+w(r)-\frac{d}{d r}\right] \\
\alpha\left[\frac{k}{r}+w(r)+\frac{d}{d r}\right] & -1+\alpha^{2} V(r)
\end{array}\right)\left(\begin{array}{l}
g(r) \\
f(r)
\end{array}\right)=\varepsilon\left(\begin{array}{l}
g(r) \\
f(r)
\end{array}\right),
$$

где $\varepsilon$ - релятивистская энергия, а $k$ - параметр спин-орбитального взаимодействия, определяемый как $k= \pm(j+1 / 2)$, где $\ell=j \pm 1 / 2$. Уравнение (3) порождает два связанных дифференциальных уравнения первого порядка для двух радиальных спинорных компонент. Исключая нижнюю компоненту, можно получить дифференциальное уравнение второго порядка для верхней компоненты. Производную первого порядка можно исключить путем общего локального унитарного преобразования

$$
\left(\begin{array}{l}
g(r) \\
f(r)
\end{array}\right)=\left(\begin{array}{cc}
\cos \rho(x) & \sin \rho(x) \\
-\sin \rho(x) & \cos \rho(x)
\end{array}\right)\left(\begin{array}{c}
\phi(x) \\
\theta(x)
\end{array}\right), \quad r=q(x)
$$

Имеем следующее условие:

$$
\frac{d q}{d x}\left[-\alpha^{2} V(r)+\cos 2 \rho+\alpha\left(w+\frac{k}{q}\right) \sin 2 \rho+\alpha \frac{d \rho / d x}{d q / d x}\right]=\text { const } \equiv \eta \neq 0 .
$$

Рассматривая случай глобального унитарного преобразования, определяемый уравнениями $q(x)=x$ и $d \rho / d x=0$, и подставляя эти соотношения в $(4)$, получаем

$$
w(r)=\frac{\alpha}{S} V(r)-\frac{k}{r}
$$

и $\eta=C+\varepsilon$, где $S=\sin 2 \rho$ и $C=\cos 2 \rho$. После применения приведенного выше преобразования и с учетом налагаемого условия радиальное уравнение Дирака (3) принимает вид

$$
\left(\begin{array}{cc}
C+2 \alpha^{2} V(r) & \alpha\left[\frac{-S}{\alpha}+\frac{\alpha C}{S} V(r)-\frac{d}{d r}\right] \\
\alpha\left[\frac{-S}{\alpha}+\frac{\alpha C}{S} V(r)+\frac{d}{d r}\right] & -C
\end{array}\right)\left(\begin{array}{c}
\phi(r) \\
\theta(r)
\end{array}\right)=\varepsilon\left(\begin{array}{c}
\phi(r) \\
\theta(r)
\end{array}\right) .
$$

Данное уравнение позволяет выразить нижнюю спинорную компоненту через верхнюю:

$$
\theta(r)=\frac{\alpha}{C+\varepsilon}\left[-\frac{S}{\alpha}+\frac{\alpha C}{S} V(r)+\frac{d}{d r}\right] \phi(r)
$$


а уравнение для верхней компоненты можно записать как

$$
\left[-\frac{d^{2}}{d r^{2}}+\frac{\alpha^{2}}{T^{2}} V^{2}(r)+2 \varepsilon V(r)-\frac{\alpha}{T} \frac{d V(r)}{d r}-\frac{\varepsilon^{2}-1}{\alpha^{2}}\right] \phi(r)=0,
$$

где $T \equiv S / C=\operatorname{tg} 2 \rho$.

Уравнение (8) представляет собой дифференциальное уравнение второго порядка для верхней спинорной компоненты типа уравнения Шредингера, в котором электростатический потенциал $V(r)$ удовлетворяет уравнению типа уравнения Риккати. Поэтому мы полагаем, что суперсимметричный подход в нерелятивистской квантовой механике можно применить для преобразования уравнения (8) в уравнение, содержащее произведение двух дифференциальных операторов первого порядка. Действительно, если выбрать дифференциальные операторы первого порядка в виде

$$
B_{ \pm}= \pm \frac{d}{d r}+\omega(r)
$$

то их произведение даст следующие гамильтонианы:

$$
\begin{aligned}
& B_{+} B_{-}=-\frac{d^{2}}{d r^{2}}+\omega^{2}(r)+\frac{d \omega}{d r} \equiv-\frac{d^{2}}{d r^{2}}+V_{1}(r), \\
& B_{-} B_{+}=-\frac{d^{2}}{d r^{2}}+\omega^{2}(r)-\frac{d \omega}{d r} \equiv-\frac{d^{2}}{d r^{2}}+V_{2}(r),
\end{aligned}
$$

где в концепции суперсимметрии в нерелятивистской квантовой механике [1], [2] функция $\omega(r)$ называется суперпотенциалом, а $V_{1}(r)$ и $V_{2}(r)$ - потенциалами-партнерами. Более того, если потенциалы-партнеры имеют одинаковую форму и различаются лишь параметрами, то говорят, что $V_{1}(r)$ и $V_{2}(r)$ - форминвариантные потенциалы. Форминвариантные потенциалы удовлетворяют уравнению [1]

$$
V_{1}\left(r, a_{0}\right)=V_{2}\left(r, a_{1}\right)+R\left(a_{1}\right)
$$

где $a_{0}$ - набор параметров, $a_{1}$ - произвольная функция от параметров $a_{0}$. Также из остатка $R\left(a_{1}\right)$ можно получить спектр собственных значений форминвариантных потенциалов [1].

В работе [8] с использованием формализма мастер-функции было показано, что оператор линейного дифференциального уравнения второго порядка общего вида и связанного с ним дифференциального уравнения математической физики можно представить в виде произведения операторов первого порядка, причем волновые функции соответствующих дифференциальных уравнений выражаются через многочлены Родригеса. В следующем разделе, используя результаты работы [9], мы найдем набор электростатических потенциалов для уравнения Дирака в терминах мастер-функции, а затем получим спинорные волновые функции в терминах представлений Родригеса для решаемых потенциалов в нерелятивистской квантовой механике. Также мы приведем спектр собственных значений уравнения Дирака для этих потенциалов. 


\section{3. ПОДХОД К РЕШЕНИЮ УРАВНЕНИЯ ДИРАКА НА ОСНОВЕ МАСТЕР-ФУНКЦИИ}

Ассоциированное дифференциальное уравнение второго порядка, записанное с помощью мастер-функции $A(x)$ и весовой функции $W(x)$, имеет следующий общий вид [8]:

$$
\begin{aligned}
A(x) \psi_{n, m}^{\prime \prime}(x) & +\frac{(A(x) W(x))^{\prime}}{W(x)} \psi_{n, m}^{\prime}(x)+ \\
& +\left[-\frac{1}{2}\left(n^{2}+n-m^{2}\right) A^{\prime \prime}(x)+(m-n)\left(\frac{A(x) W^{\prime}(x)}{W(x)}\right)^{\prime}-\right. \\
& \left.-\frac{m^{2}}{4} \frac{\left(A^{\prime}(x)\right)^{2}}{A(x)}-\frac{m}{2} \frac{A^{\prime}(x) W^{\prime}(x)}{W(x)}\right] \psi_{n, m}(x)=0,
\end{aligned}
$$

где $\psi_{n, m}(x)$ как решение в терминах многочлена Родригеса для положительного целого $n$ имеет вид

$$
\psi_{n, m}(x)=(-1)^{m} A^{m / 2}(x)\left(\frac{d}{d x}\right)^{m} \psi_{n}(x), \quad m=0,1, \ldots, n,
$$

где

$$
\psi_{n}(x)=\frac{N}{W(x)}\left(\frac{d}{d x}\right)^{n}\left(A^{n}(x) W(x)\right),
$$

a $N$ - константа. В уравнении (11) мастер-функция $A(x)$ представляет собой многочлен не выше второй степени, $W(x)$ - неотрицательная весовая функция на интервале $(a, b)$, который можно выбрать так, чтобы $A(x) W(x)$ и все ее производные равнялись нулю в точках $a, b$. Функция $\frac{1}{W(x)} \frac{d}{d x}(A(x) W(x))$ является многочленом не выше первого порядка. Нетрудно видеть, что, выбирая различные функции $A(x)$ и $W(x)$, мы можем преобразовать уравнение (11) к хорошо известным дифференциальным уравнениям второго порядка математической физики - уравнению Лагерра, гипергеометрическому уравнению, уравнению Якоби и т.д. [8], [9].

Чтобы получить уравнение Шредингера, следует исключить из уравнения (11) производную первого порядка, применив преобразование подобия. Действительно, вводя, как в работе [9], новую функцию

$$
\phi_{n, m}(r)=A^{1 / 4}(x) W^{1 / 2}(x) \psi_{n, m}(x)
$$

и выполняя замену переменных $d x / d r=\sqrt{A(x)}$, уравнение (11) можно записать в виде

$$
-\frac{d^{2}}{d r^{2}} \phi_{n, m}(r)+V_{m}(x(r)) \phi_{n, m}(r)=E(n, m) \phi_{n, m}(r)
$$

где

$$
\begin{aligned}
V_{m}(x(r))= & -\frac{1}{2}\left(\frac{A(x) W^{\prime}(x)}{W(x)}\right)^{\prime}-\frac{2 m-1}{4} A^{\prime \prime}(x)+\frac{1}{4 A(x)}\left(\frac{A(x) W^{\prime}(x)}{W(x)}\right)^{2}+ \\
& +\frac{m}{2} \frac{A^{\prime}(x) W^{\prime}(x)}{W(x)}+\frac{4 m^{2}-1}{16} \frac{\left(A^{\prime}(x)\right)^{2}}{A(x)}
\end{aligned}
$$


при этом энергетический спектр $E(n, m)$ имеет вид

$$
E(n, m)=-(n-m+1)\left[\left(\frac{A(x) W^{\prime}(x)}{W(x)}\right)^{\prime}+\frac{1}{2}(n+m) A^{\prime \prime}(x)\right] .
$$

Повышающие и понижающие операторы (9) можно определить как

$$
B_{ \pm}(m)= \pm \frac{d}{d r}+W_{m}(x(r))
$$

где суперпотенциал $W_{m}(x(r))$ выражается через мастер-функцию $A(x)$ и весовую функцию $W(x)$ :

$$
W_{m}(x(r))=-\frac{\frac{1}{2} \frac{A(x) W^{\prime}(x)}{W(x)}+\frac{2 m-1}{4} A^{\prime}(x)}{\sqrt{A(x)}} .
$$

Как было доказано в работе [9], потенциал $V_{m}(x(r))$ удовлетворяет условию форминвариантности вида

$$
\begin{aligned}
B_{+}(m) B_{-}(m) \phi_{n, m}(r) & =E(n, m) \phi_{n, m}(r), \\
B_{-}(m) B_{+}(m) \phi_{n, m-1}(r) & =E(n, m) \phi_{n, m-1}(r) .
\end{aligned}
$$

Из этих уравнений легко заметить, что при заданном $n$ оператор $B_{+}(m)$ повышает индекс $m$, а оператор $B_{-}(m)$ его понижает. Кроме того, из соотношения (16) понятно, что $E(n, m)$ обращается в нуль при $m=n+1$, поэтому, решая дифференциальное уравнение первого порядка $B_{+}(n+1) \phi_{n, n}(r)=0$, можно найти старшее состояние $\phi_{n, n}(r)$. Алгебраическое решение уравнения (15) можно получить, последовательно действуя понижающим оператором на старшие состояния [1].

Теперь, сравнивая дифференциальное уравнение (8), которое выводится из уравнения Дирака, с дифференциальным уравнением (15), мы видим, что уравнение (8) можно переписать с помощью произведения повышающих и понижающих операторов (17), задаваемых суперпотенциалом. Другими словами, мы имеем следующее соотношение между релятивистскими и нерелятивистскими параметрами:

$$
\begin{aligned}
V_{m}(r)-E(n, m) & =W_{m}^{2}(r)+\frac{d W_{m}(r)}{d r}-E(n, m)= \\
& =\frac{\alpha^{2}}{T^{2}} V^{2}(r)+2 \varepsilon V(r)-\frac{\alpha}{T} \frac{d V(r)}{d r}-\frac{\varepsilon^{2}-1}{\alpha^{2}} .
\end{aligned}
$$

Решая это уравнение, получаем следующие выражения для электростатического потенциала и релятивистской энергии:

$$
\begin{aligned}
V(r) & =-\frac{T}{\alpha}\left[W_{m}(r)+\frac{\varepsilon T}{\alpha}\right], \\
\varepsilon^{2} & =\frac{\alpha^{2}}{T^{2}+1}\left[E(n, m)+\frac{1}{\alpha^{2}}\right] .
\end{aligned}
$$

Выбирая различными способами $A(x)$ и $W(x)$, можно задать суперпотенциал и нерелятивистский энергетический спектр в соответствии с формулами (18) и (16), а затем, подставляя их в уравнение (20), найти электростатический потенциал, который 
соответствует уравнению Дирака (6). Релятивистскую энергию также можно найти из нерелятивистской энергии $E(n, m)$, используя уравнение $(21)$.

Таким образом, если мы определим электростатический потенциал $V(r)$ в терминах функций $A(x)$ и $W(x)$, то калибровочное поле $w(r)$, задаваемое равенством (5), можно выразить через функции $A(x)$ и $W(x)$. Действительно, для уравнения Дирака (6) можно получить большое число зависящих от $A(x)$ и $W(x)$ электростатических потенциалов и калибровочных полей таких, что их решение для верхней спинорной компоненты выражается через ортогональные многочлены Родригеса (12), (13). Кроме того, нижнюю спинорную компоненту волновой функции можно найти из верхней компоненты, используя (7).

В приведенном ниже примере мы довольно подробно показываем, как найти один из электростатических потенциалов при $A(x)=x$. Кроме того, в таблице приведены электростатические потенциалы для уравнения Дирака (6), которые можно найти с помощью предлагаемого метода, при различных функциях $A(x)$.

Пример. Пусть $A(x)=x$. Тогда, учитывая, что $\frac{1}{W(x)} \frac{d}{d x}(x W(x))-$ многочлен не выше первого порядка, получаем функцию $W(x)$ :

$$
W(x)=x^{\lambda} e^{-\gamma x}, \quad x=\frac{r^{2}}{4}, \quad 0<x<+\infty,
$$

где $\lambda>-1$ и $\gamma>0$. Используя (18) и (16), получаем суперпотенциал и нерелятивистскую энергию:

$$
W_{m}(r)=-\left(\lambda+m-\frac{1}{2}\right) \frac{1}{r}+\frac{\gamma}{4} r, \quad E(n, m)=\gamma(n-m+1) .
$$

Подстановка этих формул в (20), (21) дает следующие соотношения для электростатического потенциала и релятивистской энергии:

$$
\begin{aligned}
V(r) & =-\frac{T}{\alpha}\left[-\left(\lambda+m-\frac{1}{2}\right) \frac{1}{r}+\frac{\gamma}{4} r\right]-\frac{\varepsilon T^{2}}{\alpha^{2}}, \\
\varepsilon^{2} & =\frac{\alpha^{2}}{T^{2}+1}\left[\gamma(n-m+1)+\frac{1}{\alpha^{2}}\right] .
\end{aligned}
$$

Подставляя эти выражения в уравнение (8), получаем следующее дифференциальное уравнение для верхней спинорной компоненты $\phi_{n, m}(r)$ :

$$
\begin{aligned}
& -\frac{d^{2}}{d r^{2}} \phi_{n, m}(r)+\left[\frac{\gamma^{2}}{16} r^{2}+\left(\lambda+m+\frac{1}{2}\right) \times\right. \\
& \left.\times\left(\lambda+m-\frac{1}{2}\right) \frac{1}{r^{2}}-\frac{\gamma}{2}(\lambda+m-1)\right] \phi_{n, m}(r)=\gamma(n-m+1) \phi_{n, m}(r) .
\end{aligned}
$$

Итак, мы получили уравнение Шредингера с потенциалом трехмерного гармонического осциллятора с энергетическим спектром вида $\gamma(n-m+1)$. Нетрудно видеть, что этот потенциал имеет форминвариантную симметрию, и его можно представить в виде операторного произведения повышающих и понижающих операторов:

$$
\begin{aligned}
B_{+}(m) B_{-}(m) \phi_{n, m}(r) & =\gamma(n-m+1) \phi_{n, m}(r), \\
B_{-}(m) B_{+}(m) \phi_{n, m-1}(r) & =\gamma(n-m+1) \phi_{n, m-1}(r),
\end{aligned}
$$




\begin{tabular}{|c|c|c|c|c|c|c|c|}
\hline 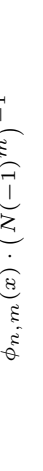 & 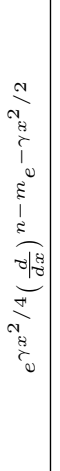 & 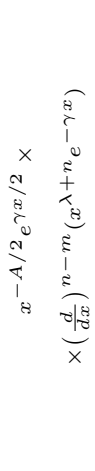 & 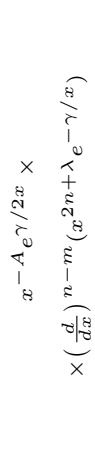 & 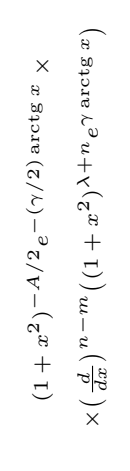 & 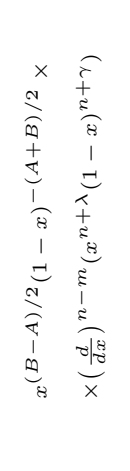 & 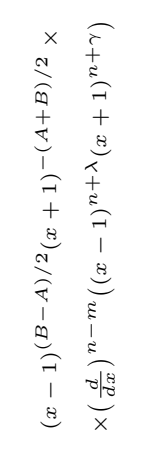 & 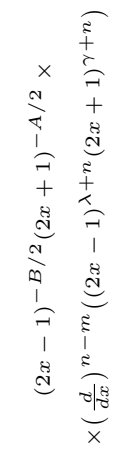 \\
\hline 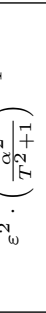 & 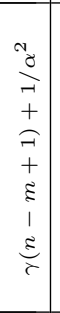 & 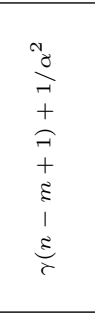 & 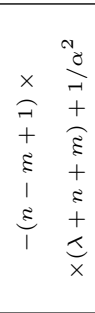 & 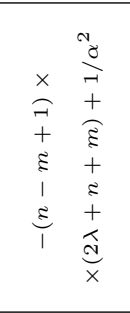 & 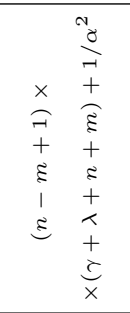 & 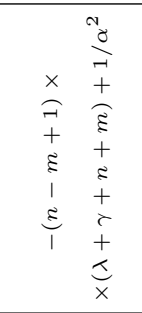 & 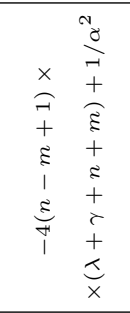 \\
\hline $\begin{array}{l}\frac{1}{1} \\
\frac{10}{1} \\
\frac{1}{5} \\
\frac{5}{5}\end{array}$ & 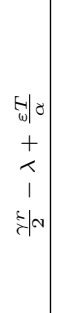 & 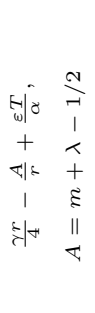 & 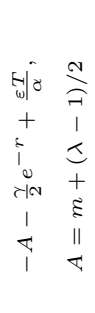 & 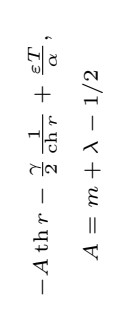 & 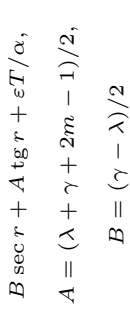 & 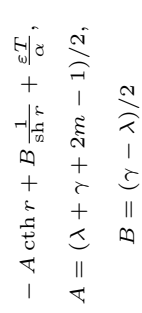 & 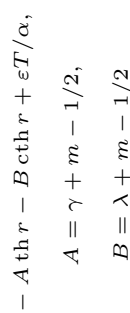 \\
\hline $\begin{array}{l}\bar{\theta} \\
\bar{B}\end{array}$ & 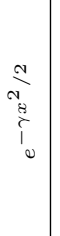 & 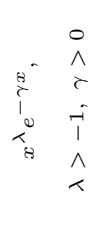 & 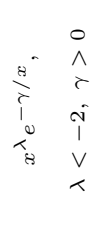 & 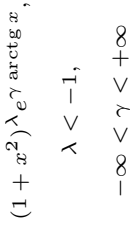 & 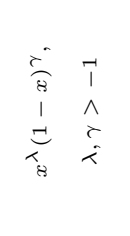 & 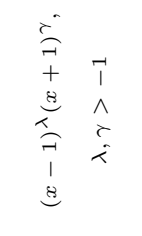 & 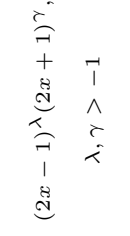 \\
\hline 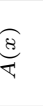 & $r$ & 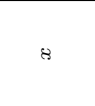 & ${ }_{B}^{N}$ & $\begin{array}{l}{ }_{\&}^{8} \\
+ \\
+\end{array}$ & $\begin{array}{l}\vec{\theta} \\
1 \\
\vec{\theta} \\
\end{array}$ & 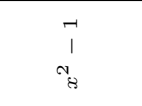 & $\begin{array}{l}\overrightarrow{1} \\
1 \\
N_{y} \\
y\end{array}$ \\
\hline 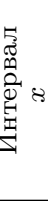 & $\begin{array}{l}8 \\
+ \\
\vee \\
8 \\
\vee \\
8 \\
1\end{array}$ & $\begin{array}{l}8 \\
+ \\
v \\
8 \\
v \\
0\end{array}$ & $\begin{array}{l}8 \\
+ \\
V \\
8 \\
V \\
0\end{array}$ & $\begin{array}{l}8 \\
+ \\
\vee \\
8 \\
\vee \\
8 \\
1\end{array}$ & $\begin{array}{l}\vec{V} \\
\forall \\
V \\
0\end{array}$ & $\begin{array}{l}\vec{v} \\
8 \\
v \\
\overrightarrow{1}\end{array}$ & 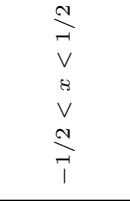 \\
\hline$\underset{్}{E ్ ~}$ & $\begin{array}{l}\bar{y} \\
\text { a } \\
1 \\
t\end{array}$ & 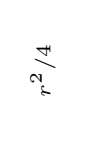 & to & $\begin{array}{l}\text { के } \\
\text { n. } \\
\| \\
\&\end{array}$ & $\begin{array}{l}\stackrel{N}{E} \\
\stackrel{\Xi}{n} \\
+ \\
=\end{array}$ & fi & $\begin{array}{l}\stackrel{N}{\mathrm{I}} \\
\text { I } \\
\text { d }\end{array}$ \\
\hline
\end{tabular}


где

$$
B_{ \pm}(m)= \pm \frac{d}{d r}+\left[\frac{\gamma}{4} r-\left(\lambda+m-\frac{1}{2}\right) \frac{1}{r}\right] .
$$

Теперь, вводя новую функцию согласно формуле (14) как

$$
\phi_{n, m}(r)=x^{\lambda / 2+1 / 4} e^{-\gamma x / 2} \psi_{n, m}(x)
$$

и выполняя замену переменных $d x / d r=\sqrt{x}$, можно преобразовать уравнение $(22)$ к ассоциированному дифференциальному уравнению

$$
\begin{aligned}
x \frac{d^{2}}{d x^{2}} \psi_{n, m}(x) & +((\lambda+1)-\gamma x) \frac{d}{d x} \psi_{n, m}(x)+ \\
+ & {\left[\gamma\left(n-\frac{m}{2}\right)-\frac{m}{4}(m+2 \lambda) \frac{1}{x}\right] \psi_{n, m}(x)=0, }
\end{aligned}
$$

где $\psi_{n, m}(x)$ определяется в соответствии с (12) как

$$
\psi_{n, m}(x)=(-1)^{m} x^{m / 2}\left(\frac{d}{d x}\right)^{m} \psi_{n}(x),
$$

а $\psi_{n}(x)$ удовлетворяет дифференциальному уравнению Лагерра и имеет следующее представление Родригеса:

$$
\psi_{n}(x)=N x^{-\lambda} e^{\gamma x}\left(\frac{d}{d x}\right)^{n}\left(x^{\lambda+n} e^{-\gamma x}\right) .
$$

Таким образом, мы нашли решение уравнения Дирака (3) с электростатическим потенциалом (20) и калибровочным полем (5). Другими словами, мы показали, что как релятивистская энергия (21), так и спинорная волновая функция удовлетворяют двухкомпонентному радиальному уравнению Дирака (1). Кроме того, ясно, что верхняя спинорная компонента $\phi_{n, m}(r)$ записывается в терминах многочлена Родригеса (23), а нижняя получается из старшего состояния с помощью уравнения (7).

При другом выборе функций $A(x)$ и $W(x)$, проделав аналогичные выкладки, мы можем получить решения уравнения Дирака с другими скалярными потенциалами. Результаты приведены в таблице.

\section{4. ЗАКЛЮЧЕНИЕ}

Для вывода дифференциального уравнения второго порядка типа уравнения Шредингера для верхней спинорной компоненты мы следовали процедуре решения уравнения Дирака в электромагнитном поле со сферически-симметричными потенциалами. Мы показали, что полученное уравнение можно преобразовать к уравнению Шредингера с форминвариантными потенциалами. Используя подход мастерфункции при различных видах электростатического потенциала и релятивистской энергии, записываемых через мастер-функции $A(x)$ и весовые функции $W(x)$, мы нашли решения уравнение Дирака и получили спинорные волновые функции в терминах многочленов Родригеса, соответствующие каждому случаю. 


\section{Список литературы}

[1] F. Cooper, A. Khare, U. Sukhatme, Phys. Rep., 251:5-6 (1995), 267-385.

[2] A. B. Balantekin, Phys. Rev. A, 57:6 (1998), 4188-4191.

[3] J. W. Dabrowska, A. Khare, U. P. Sukhatme, J. Phys. A, 21:4 (1988), L195-L200.

[4] W. Greiner, Relativistic Quantum Mechanics. Wave Equations, Theoretical Physics: Text and Exercise Books, Springer, Berlin, 1990.

[5] A. D. Alhaidari, Phys. Rev. Lett., 87:21 (2001), 210405, 4 pp., arXiv: hep-th/0112001.

[6] M. Moshinsky, A. Szczepaniak, J. Phys. A, 22:17 (1989), L817-L819.

[7] A. D. Alhaidari, J. Phys. A, 35:29 (2002), 6207-6216.

[8] M. A. Jafarizadeh, H. Fakhri, Phys. Lett. A, 230:3-4 (1997), 164-170.

[9] M. A. Jafarizadeh, H. Fakhri, Ann. Phys., 262:2 (1998), 260-276.

Поступила в редакцию 25.10.2009 